\title{
A method of gate hoist gear fault diagnosis based on chaotic oscillator
}

\author{
Ying Wang ${ }^{1,2, a}$, Yourong $\mathrm{Li}^{1, \mathrm{~b}}$ and Xianwang $\mathrm{Fu}^{2, \mathrm{c}}$ \\ ${ }^{1}$ The Key Laboratory for Metallurgical Equipment and Control of the Ministry of Education, \\ Wuhan University of Science and Technology, Wuhan 430081, China \\ ${ }^{2}$ Hubei Key Laboratory of Hydroelectric Machinery Design \& Maintenance, China Three \\ Gorges University, Yichang 443002, China

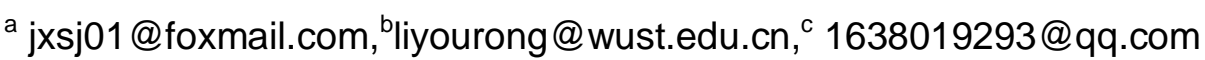

Keywords: gear fault; gate hoist; Chaotic Oscillator; diagnosis

Abstract. By taking the gear box of a ship lock's hoisting machine as the research object, because of the vibration signal of a fault gate has the characteristics of both randomness and non-stationary, the chaos oscillator detection theory is introduced into the diagnosis for gear fault. Research the dynamic behavior of the gear under different condition of various revolving speed and fault parameters based on the vibration model, analyze the variations of the change of phase trajectory of the gear oscillator by through the bifurcation diagram, phase diagram, power spectrum and time histories, the fault of the gear could be diagnosed. The method has been verified effectively to extract the early fault of gear box by inspection, solved the poor effect by spectrum analysis as the lower frequency response because of the shock by the low speed, the failure point, a new method for the low speed and heavy duty gear box fault diagnosis has been proposed

\section{Introduction}

The gate hoist gearboxes are important equipment in hydropower project production operation, the working environment of the system is variable, and the fault impact interval is longer, when it runs under different working conditions or in weak fault cases, it is difficult to find out the potential failure about crack or abrasion of the gear box part, to analyze its dynamic performance has important effect to the stable operation of the equipment. In this paper, based on the gear fault vibration mathematical model, using the theory of chaotic oscillator detection, analyzing the mechanism of the dynamic behavior change of gear fault from two aspects that the last perturbation of different conditions or different fault degree with the gear, discussing the phase difference under the different condition of gear oscillator, attempting to realize the fault diagnosis analysis of gate hoist gear.

\section{The diagnosed principle of chaotic oscillator}

Chaotic oscillator diagnosis method is based on the theory of chaotic oscillator detection. The detection principle is derived from the control of chaos. The so-called chaos control is to suppress the chaos, which is used to carry on the periodic disturbance to the system. To achieve the purpose of system from unsteadiness to stabilize. Chaotic oscillator detection mainly uses the sensitivity of chaotic system to the equation parameters. So that the system occur to chaos phase change of the critical state even when the weak signal amplitude is very small. Then the weak periodic signal will be detected by identifying the system state. 
Chaos theory, which is an emerging discipline, even though have been developed for nearly half a century, there is no accurate definition. A generally accepted Yorke definition was been introduced in this paper.

Assuming that $f(\mathrm{x})$ be a continuous map of $I \subset R$ to itself, and there are 3 periodic points of $f(\mathrm{x})$, let $\mathrm{n}$ be a positive integer, and $\mathrm{n}$ periodic points contained with $f(\mathrm{x})$. There is an uncountable subset satisfied:

(1) No periodic point existed in $S$;

(2) Let $x, y \in S(x \neq y)$ :

$\liminf _{n \rightarrow \infty}\left|f^{n}(x)-f^{n}(y)\right|=0$

$\limsup _{n \rightarrow \infty}\left|f^{n}(x)-f^{n}(y)\right|>0$

(3) Let $x \in S$ and $y$ is the periodic point of $f$, if $\limsup _{n \rightarrow \infty}\left|f^{n}(x)-f^{n}(y)\right|>0$, then $f$ is chaotic at $s$

According to the above definitions, when $f(\mathrm{x})$ be self-mapping of interval $I$, if there is a periodic point equals 3 , positive integer periodic points must be existed, chaotic phenomena be called.

Due to the both complicated and rich of the internal structure of chaos, it is necessary to analyze its connotation from a number of different aspects. Numerical method, analytical method, statistical description method and experimental method are the common method at present. In this paper, by adopting the method of numerical analysis of chaotic oscillator, applying the chaotic oscillator detection method to gear fault diagnosis, Reference for chaos control in perturbation theory, with the aid of the method when gear oscillator system are in a state of chaos and quasi periodic critical, fault signal of continuous perturbation incentives will change the behavior of the system dynamics to diagnose the different failure modes of the gears. And the main research methods contain: 1) phase diagram (space trajectory); 2) time histories diagram (time domain waveform); 3) Poincare section; 4) power spectrum.

\section{Analysis of gear fault diagnosis based on chaotic oscillator}

As the key components in the hoist, gear is often keep station of continuous or start-stop working repetitively, the speed have important effects on the working condition of gear system. Consequently, it's necessary to discuss the changing in dynamic behavior at different rotary speed and different fault level.

Three-stage gear low speed and heavy-duty gear box is used in the horizontal type gear reducer in Mechanical Hoist of the hydropower station gate. According to the analysis to the operation feature and testing technique, testing in site at scheduled overhaul in 2012, collecting vibration signals before and after maintenance, verifying the efficiency and applicability of testing technology. The location of sensors are shown in figure 1, two sensors placed on the tops of the gear box, and the other four sensors arrangement on the four rolling bearing housings respectively, they are used for collecting the fault signal of the gears and the four rolling bearings, the gate hoist gear box opening and closing vibration signals has been collected three times before maintenance and three times after maintenance. 


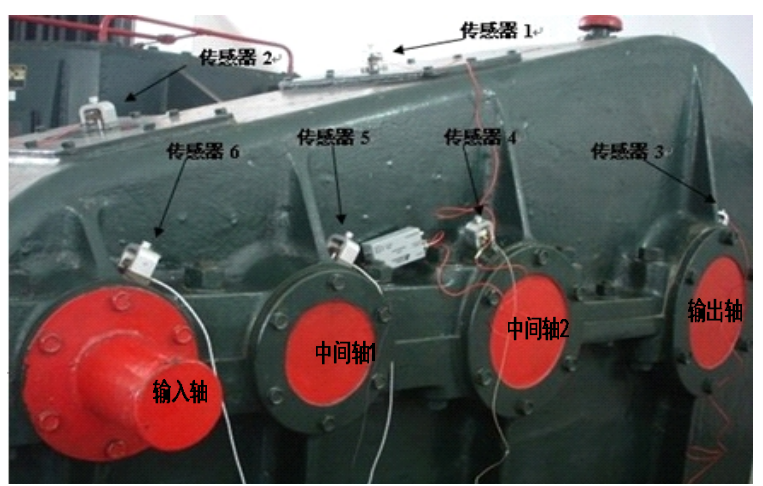

Fig.1. The arrangement of sensors

\section{Dynamics analysis with the normal state of gear}

Solving the equation whose variable is the speed parameter, the map of bifurcation diagrams for the system is obtained, for the accurate analysis of motion state, take different speed conditions with the gear meshing angular frequency equals $0.6,1.5,1.7$ and 2.0, solving the equation respectively, phase diagram and displacement power spectrum diagram of the system are received just as figure (2) and figure (3). The motion state of the system under different parameter in figure (2) could be verified by the motion waveform of single period, chaos, period-doubling and quasi-period of time domain waveform just as figure (3). For the gear systems, the using of phase change gear vibration subsystem to detect faint change of running status of gear is very sensitive, it can be applied to the fault diagnosis.

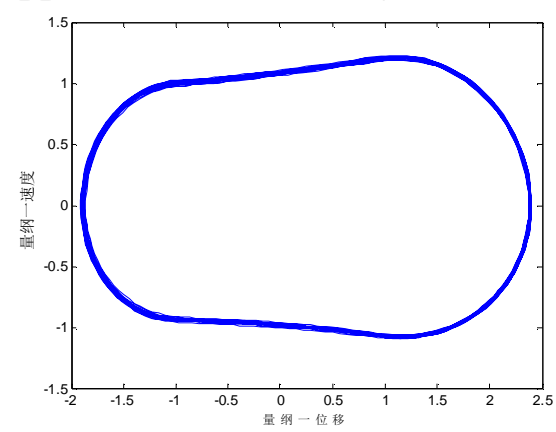

(a) phase diagram of $\omega=0.6$

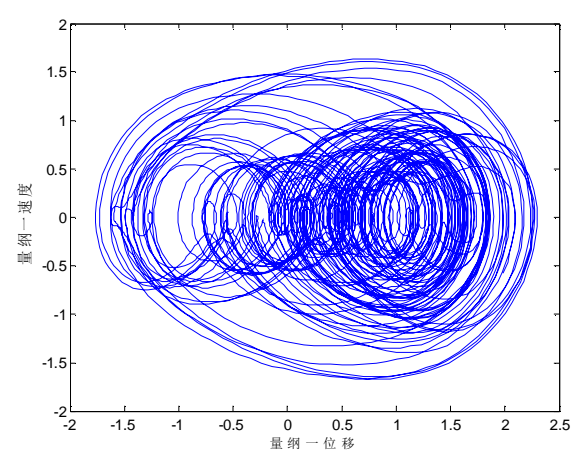

(c) phase diagram of $\omega=1.5$

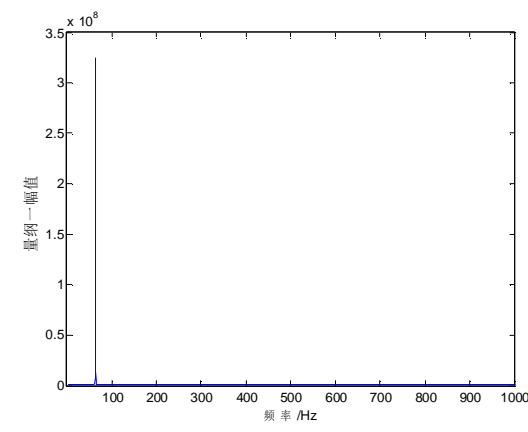

(b) power spectrum of $\omega=0.6$

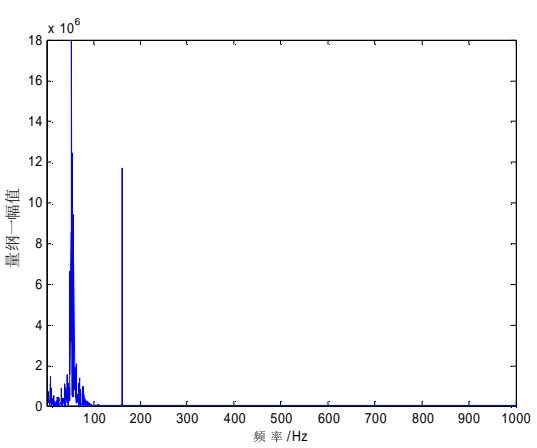

(d) power spectrum o $\omega=1.5$ 


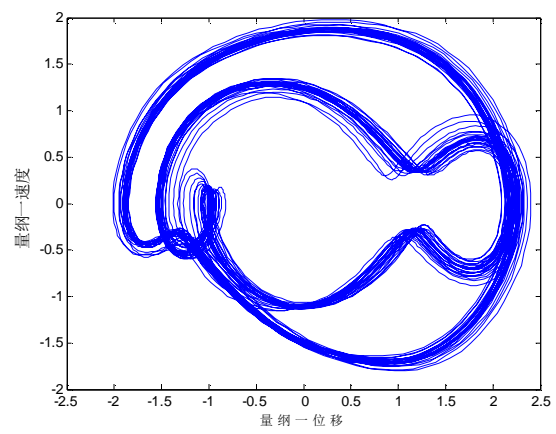

(e) phase diagram of $\omega=1.7$

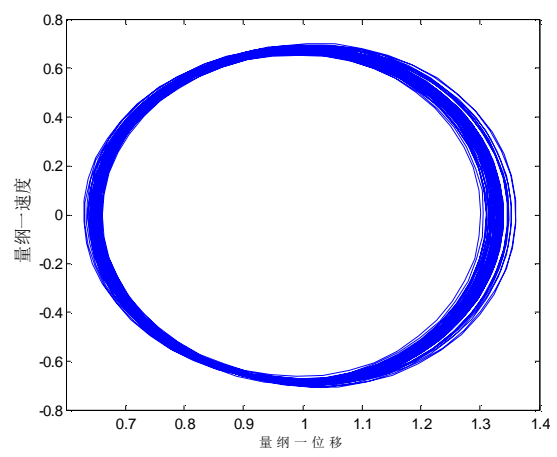

(g) phase diagram of $\omega=2.0$

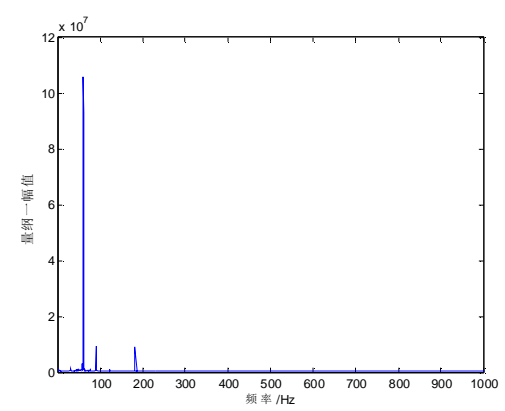

(f) power spectrum o $\omega=1.7$

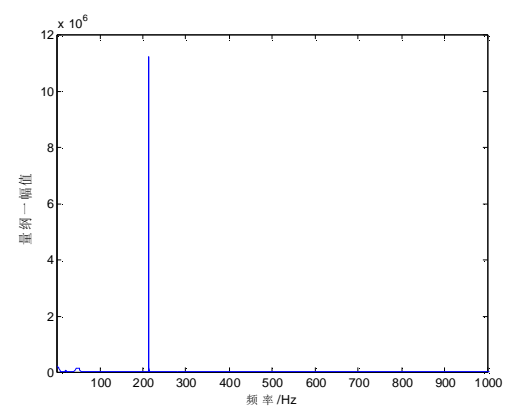

(h) power spectrum o $\omega=2.0$

Fig.2 The phase diagram and power spectrum chart of the normal gear

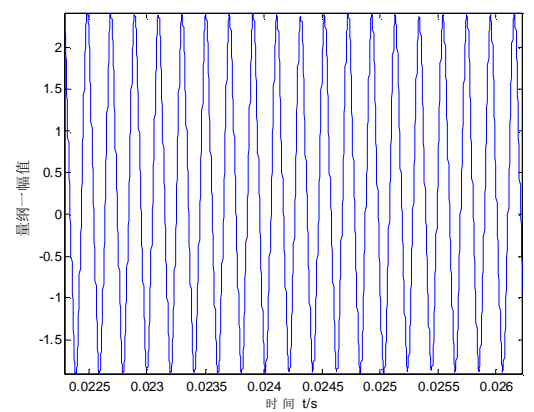

(a) time histories with $\omega=0.6$

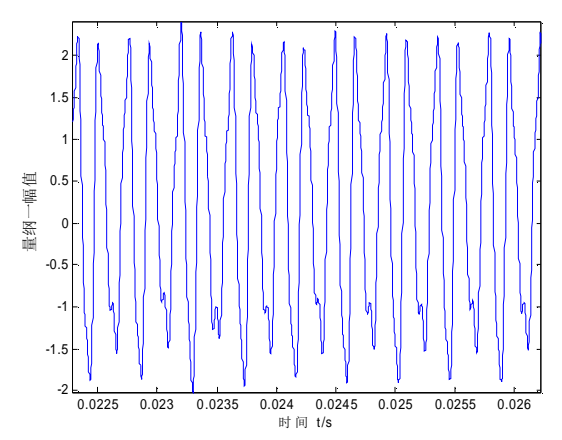

(c) time histories with $\omega=1.7$

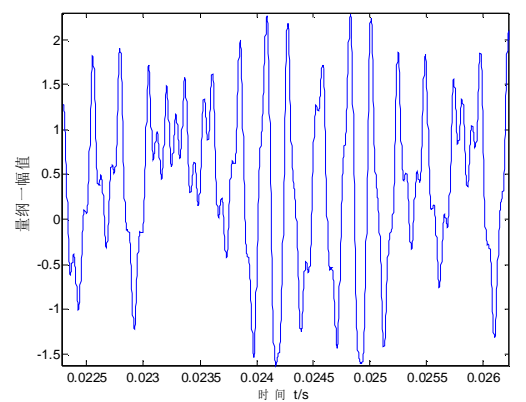

(b) time histories with $\omega=1.5$

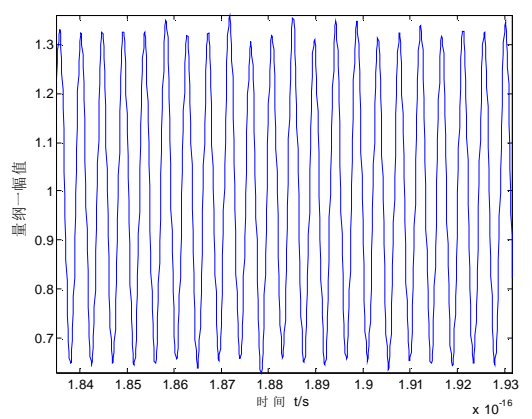

(d) time histories with $\omega=2.0$

Fig.3 Time histories of normal gear

\section{The fault diagnosis analysis of the cracked gear}

(1) In the crack of the dedendum is mild (let the crack depth of the gear ), doing the system dynamics analysis under the condition of different parameters of its speed. The map of bifurcation diagrams for the system is obtained as shown in figure 4 , take different speed conditions with the gear meshing angular frequency equals $0.31,0.48$ and 0.65 , solving the equation respectively, phase 
diagram and power spectrum diagram of the system are received just as figure (6).From the two figures, it shows that with the increase of rotational speed, the system turn into the quasi periodic motion after multiple times of the periodic motion, so it is sensitive to the crack fault parameters, the chaos state did not appear, the period of the disturbance of the fault is very outstanding and persistent

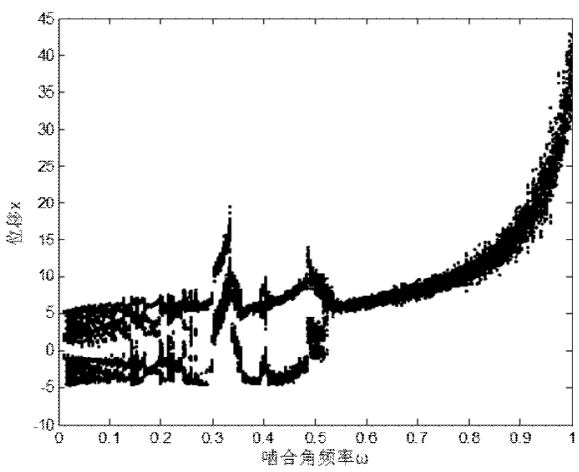

Fig.4 Bifurcation diagram of the crack gear under different speed

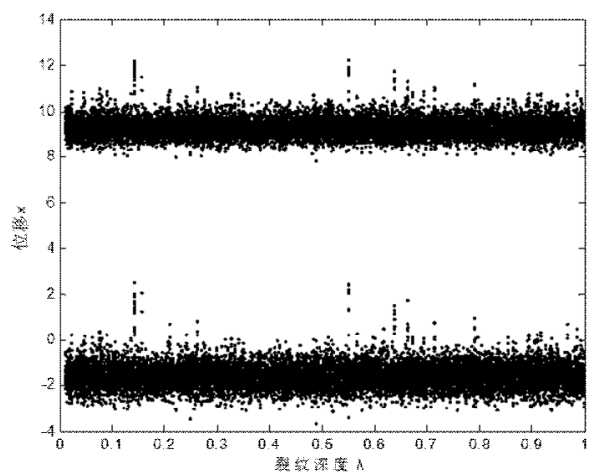

Fig.5 Bifurcation diagram of the crack gear under different crack depth

(2) Invariable turning speed (let $\omega=0.48$ ), doing the system dynamics analysis under the condition of different parameters of the crack depth. The value of the crack depth $\lambda$ range from 0.1 to 1 , solving the crack fault equation of the system, the map of bifurcation diagrams for the system is obtained as shown in figure 5, then draw the phase diagram and power spectrum diagram of the system as figure 6 (a)and(b).

From figure 5 and figure 6 (a) and (b), when the keeps invariant, With the increase of crack depth, there is no obvious change about attractor trajectories of the system, bifurcation phenomenon does not appear, it Always stay in the initial motion. Decreased the to 0.31 and increased it to 0.65 respectively, then aggrandized the degree of the crack fault gradually, received the phase diagram of the system as figure 6(c) and 6(e) by solving the equation, From the figures, we can find that there is no obvious change about the attractor trajectories, the topological structure and the measure of phase diagram, the corresponding power spectra are discrete spectral lines, it showed that when the crack of gear appeared, the condition of the system motions entered into periodic motion immediately, and the parameters with the degree of crack fault have little effects on the dynamic behavior of the nonlinear gear system, the effect of diagnostic is not obvious.

\section{The fault diagnosis analysis of the uniform weared gear}

(1) Mild wear with the gear (let the ratio between gear back lash and wear loss $s=0.1$ ), analyze the dynamic behavior of the system under the different condition with various speed parameter. When the gear system failure generated, the corresponding fault parameters varied with the change of the fault, took the slight wear of the gear as an example, analyze the dynamic behavior under different speed condition of the failure gear, solving the wear fault

$$
\text { equation } M_{e} \ddot{X}(t)+C_{g} \ddot{X}(t)+K_{z}(t) f(X(t))=F_{s}+K_{z}(t) e_{m}(t) \text {, the bifurcation diagram of }
$$

uniform wear fault of the gear was obtained as figure 7, then let the $\omega=0.05,0.1,0.18$ and 0.4 , draw their phase diagram as figure 8 , the corresponding power spectrum and process diagram are 


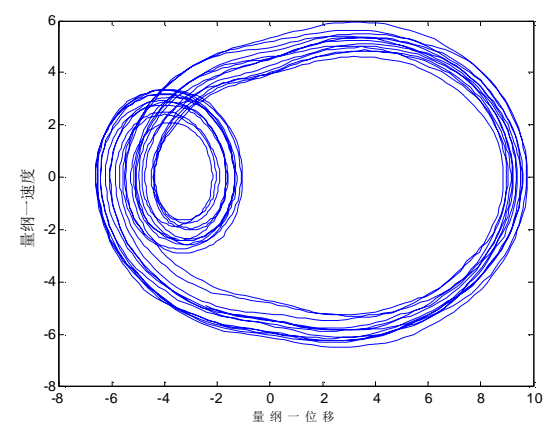

(a) phase diagram of $\omega=0.48$ and $\lambda=0.1 \sim 1$

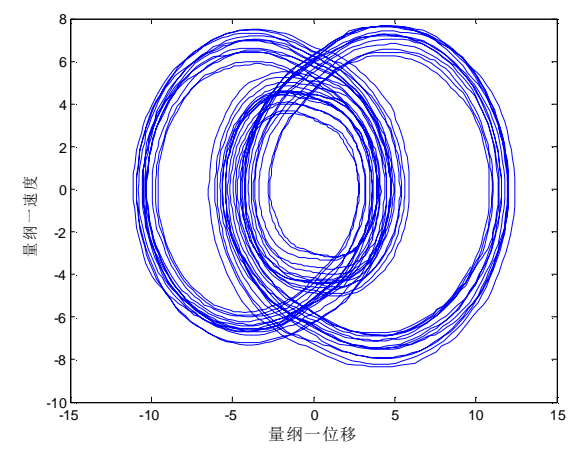

(c) phase diagram of $\omega=0.31$ and $\lambda=0.1 \sim 1$

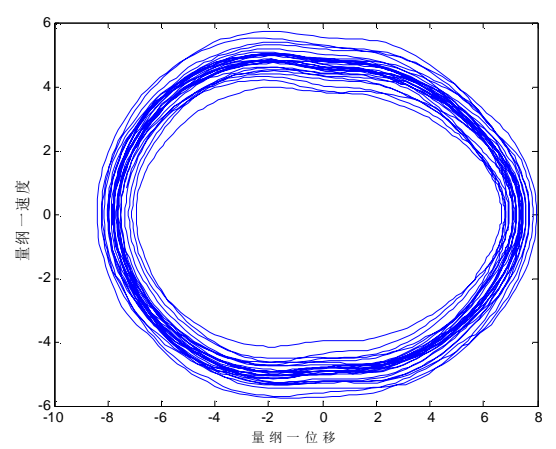

(e) phase diagram of $\omega=0.65$ and $\lambda=0.1 \sim 1$

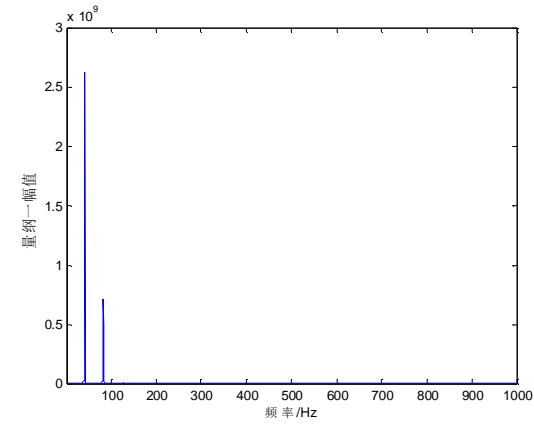

(b) power spectrum of $\omega=0.48$ and $\lambda=0.1 \sim 1$

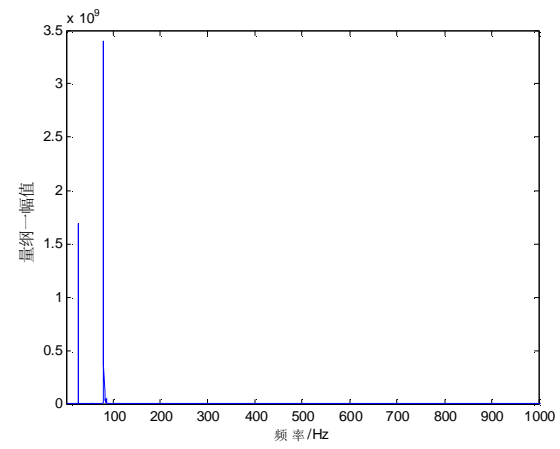

(d)power spectrum of $\omega=0.31$ and $\lambda=0.1 \sim 1$

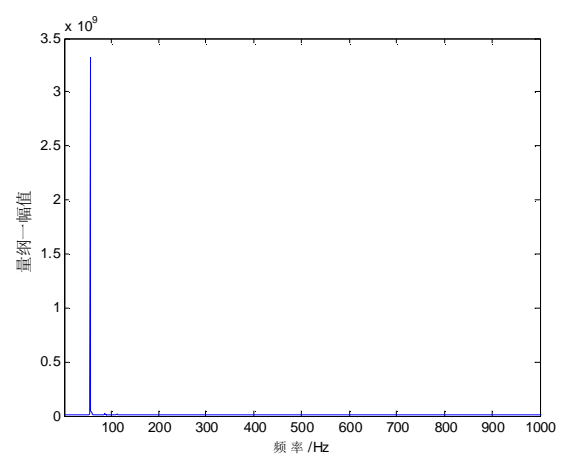

(f) power spectrum of $\omega=0.65$ and $\lambda=0.1 \sim 1$

Fig.6 Phase diagram and power spectra of gear crack fault

ignored here. Compared with figure 7and 9, it shows that when the gear is mild wear, with the increase of rotational speed, the attractor of system change from initial chaotic state to cycle time period-doubling and quasi periodic condition, increase speed continued, it turns into big chaos, when $\omega$ exceed a certain threshold, it stabilize in a single cycle motion ultimately.

Compared with the normal state, the effect of disturbance caused by the gear fault may be huge on the system's attractor trajectory under the small rotate speed. Even if rotate speed of gear is within the actual allowed range, it will lead to a series of changes of state from period-doubling to chaos and then to period. The topological structure of the phase diagram is also changed, and the system enters a single cycle when the rotation speed increases to a certain extent. Compared with the crack fault, the uniform wear failure makes system into a stable movement state at a low speed, and arise chaos movement within a certain rotate speed. In consequence, at the same operating speed, it's easy to diagnose if the gear appears cracks fault or uniform wear failure by corresponding regularity of system. 


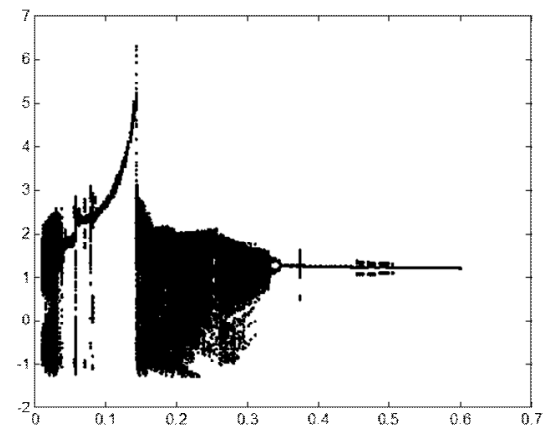

Fig.7 Bifurcation diagram of the even wear fault gear under different working conditions

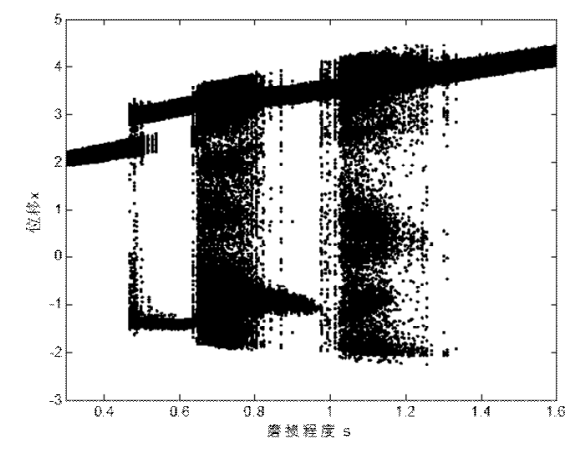

Fig. 8 The bifurcation diagram of fault gear under different attrition rate

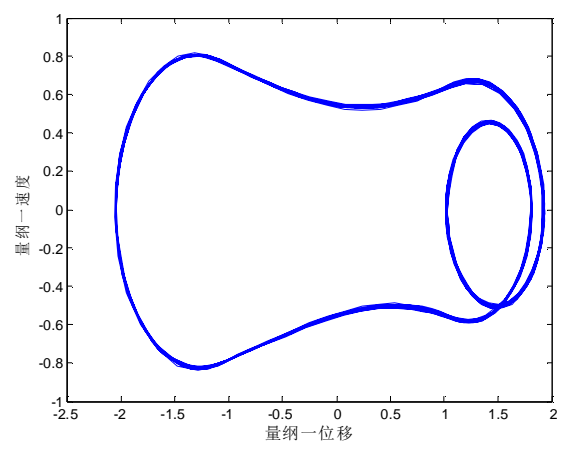

(a) phase diagram of $\omega=0.05$

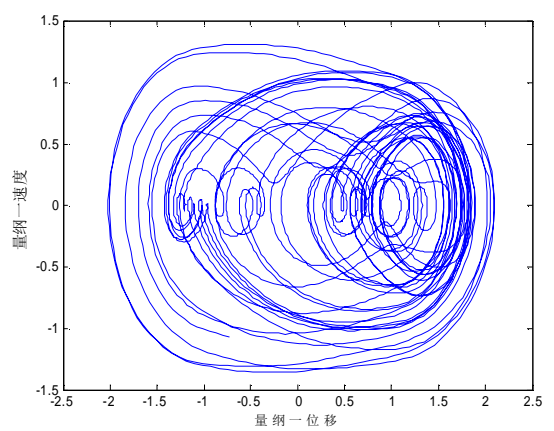

(c) phase diagram of $\omega=0.18$

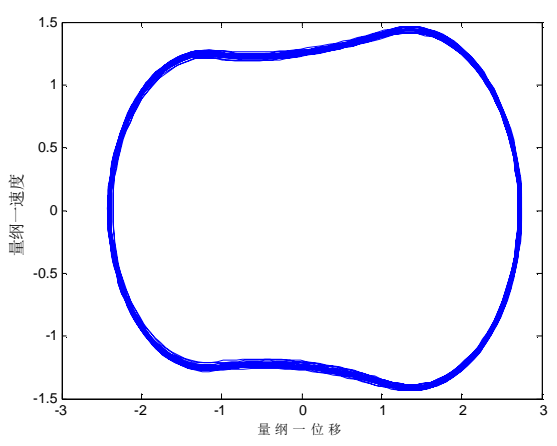

(b) phase diagram of $\omega=0.1$

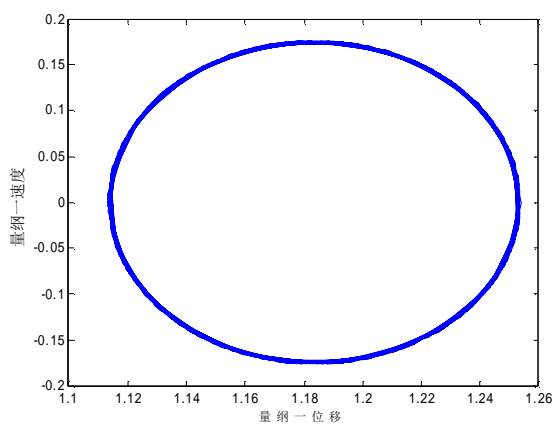

(d) phase diagram of $\omega=0.4$

Fig.9 The phase diagram of wear failure gear

(2) The constant rotor speed (set $\mathrm{w}=0.05$ ), thinking about the dynamic analysis of the different condition of the wear failure. According to the mentioned calculation method, make uniform wear failure extent within the scope of increased gradually from 0.3 to 1.6. getting the bifurcation diagram of the wear failure by the wear gear fault equation in figure 9 , and choose the different parameters to draw the system phase diagram and power spectrum diagram as shown in figure 10 , such as $s$ is $0.5,07,1.1,1.4$.

From the comparison of the two figures 8 and 10, it can be seen that from a quasi-periodic movement, gear system has experienced twice cycle and chaotic motion before it stabled in quasi-periodic motion under conditions of increasing the degree of wear. The topology of the phase diagram has a series of changes and the power spectrum of frequency amplitude is gradually increased. Dynamical behavior of the system for uniform wear parameter has a higher degree of sensitivity and motion increases with the fault degree showing a strong variation: The initial and 
final states are quasi-periodic motion. In the middle, the period-doubling transitioned to chaotic motion of two bifurcations and the twice transitionary catastrophe from chaos to period-doubling. It unlike any other parameters of the system transformation, the fault diagnosis method based on dynamic analysis is better on diagnosis effect of wear degree.

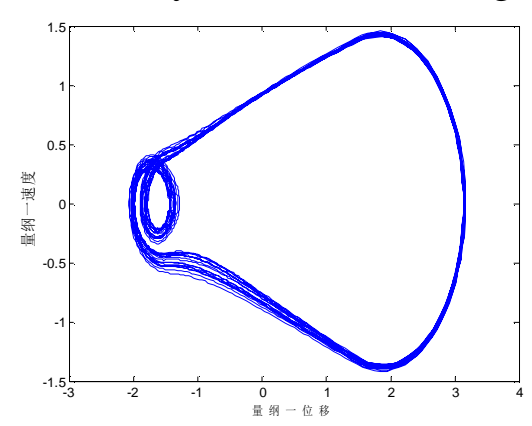

(a) phase diagram of $s=0.5$

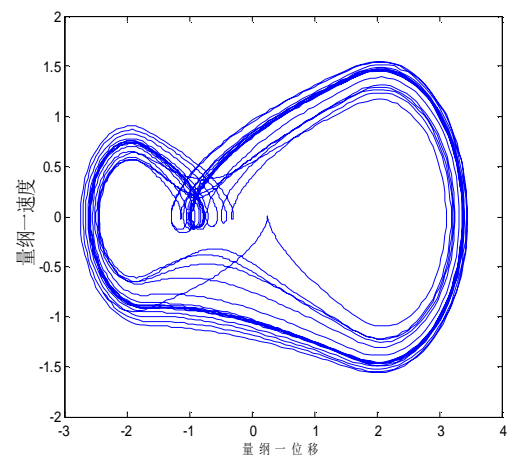

(c) phase diagram of $s=0.7$

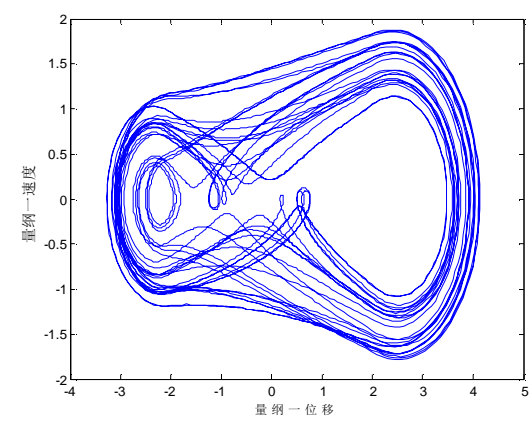

(e) phase diagram of $s=1.1$

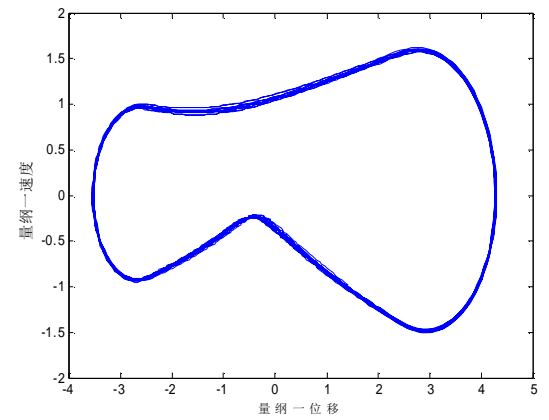

(g) phase diagram of $s=1.4$

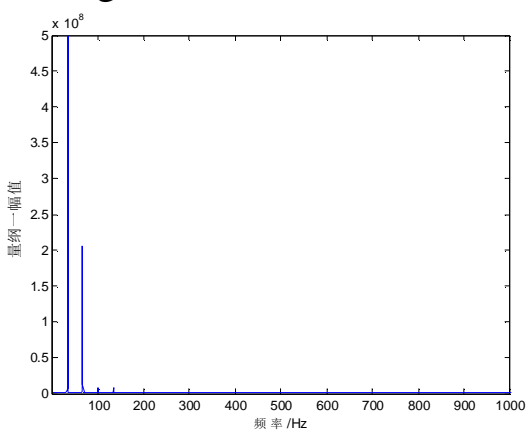

(b) power spectrum of $s=0.5$

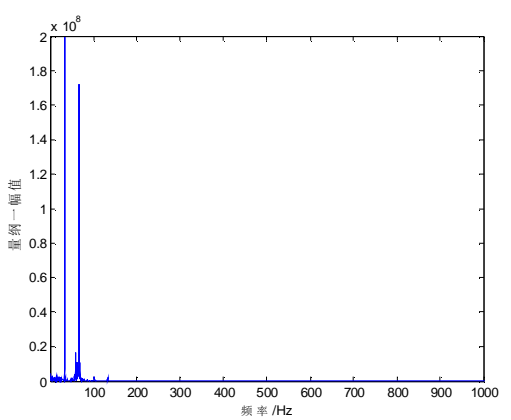

(d) power spectrum of $s=0.7$

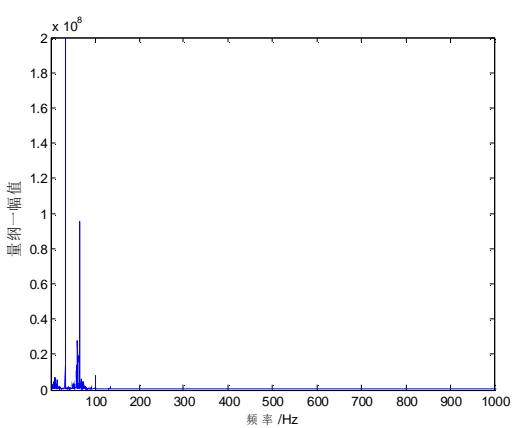

(f) power spectrum of $s=1.1$

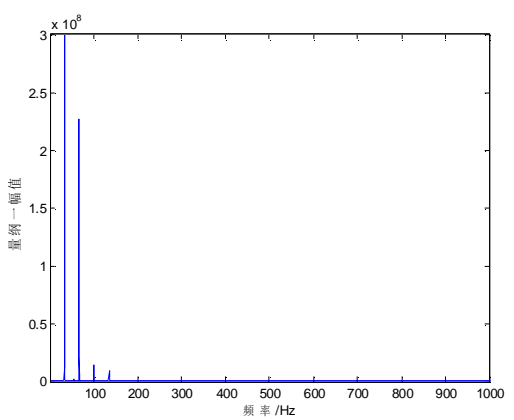

(h) power spectrum of $s=1.4$

Fig. 10 The phase diagram and power spectrum of wear failure gear 


\section{Conclusion}

According to the vibration model, the vibration displacement and velocity signal of the system are obtained, and the trajectory of the system is made. Referring to perturbation theory of the chaotic system, the dynamic behaviors of the gear system under different operating conditions and different failure modes are studied. The results show that with the increase of the degree of gear failure, the phase diagram attractors of the crack fault has little change, the crack depth has little effect on the movement of the system, and the diagnosis effect is not obvious. However, with the increase of the speed, the crack fault system of the gear changes from the periodic motion to the periodic motion. When the rotation speed is constant, the motion of the uniform wear fault system is started from the quasi periodic motion, after two times of double-periodic and chaotic motion, finally stable in quasi periodic motion state. Under different speed conditions, the system is changed from Chaos to quasi periodic, double periodic and chaotic motion, and finally stable in a single period state. The topological structure of the phase diagram is constantly changing, that is, dynamic phase transformation of the gear oscillator is different under different working conditions parameters and different fault parameters, which can diagnosis and analysis the gear fault.

After the maintenance of a floodgate hoist gear box, the maintenance results and analysis results are in accordance with the conclusion. After adjusting the bearing clearance and locating the bearing, the phenomenon before maintenance disappeared, which proved this method is feasible for the fault diagnosis of large gear box. The development process of the failure can be predicted by this method to find the early failure, to take measures to avoid major accidents, to reduce the risk of accidents.

\section{References}

[1] Jin-ming Lu, Fan-Sen Meng, Gear fault diagnosis based on ensemble empirical mode and instantaneous energy density spectrum, Journal of Jiangsu University of Science and Technology (Natural Science Edition). pp. 262-265, March, 2012.

[2] Chong-feng Cao, Shi-xi Yang, De-noising method for non-stationary vibration signals of large rotating machineries based on ensemble empirical mode decomposition. Journal of Vibration and Shock, vol. 28, no 9. pp: 33-38, Sep, 2009.

[3] Yue Li, Bao-jun Yang, An introduction to the chaotic oscillator detection, first ed., Peking, 2004

[4] Qin Cui, Weak Signal Detection Based on Chaos Theory, first ed., Harbin, 2008

[5] Henry, B, Lovell, N. and Camacho, F. Nonlinear Dynamics Time Series Analysis, in Nonlinear Biomedical Signal Processing: Dynamic Analysis and Modeling, Volume 2 (ed M. Akay), John Wiley \& Sons, Inc., Hoboken, NJ, USA, 2000

[6] Li T, Yorke J. Period three implies chaos. Amer. Math. Monthly, 1975, 82(10):985-992

[7] Yu-shu Chen, Yun Tang, Modern Analytic Method of Nonlinear Dynamic, Peking, 2000 